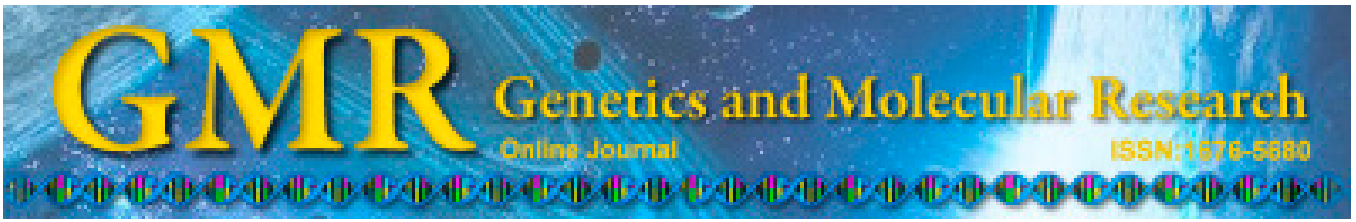

\title{
Screening relevant genes of tolerance to low phosphorus in maize using cDNA-amplified fragment length polymorphism
}

\author{
H.Y. Jiang, Z. Li, J. Zhao, Q. Ma, B.J. Cheng and S.W. Zhu \\ Key Lab of Crop Biology, School of Life Sciences, Anhui Agricultural University, \\ Hefei, China \\ Corresponding author: S.W. Zhu \\ E-mail: zhusuwen@126.com \\ Genet. Mol. Res. 14 (2): 5731-5741 (2015) \\ Received June 13, 2014 \\ Accepted December 3, 2014 \\ Published May 29, 2015 \\ DOI http://dx.doi.org/10.4238/2015.May.29.5
}

\begin{abstract}
Soil contains a large amount of phosphorus, but plants cannot absorb most of this phosphorus effectively. Low inorganic phosphorus has been singled out as a major constraint that leads to a perpetually low Zea mays (maize) grain yield. The fundamental approach to solving this problem is to screen new genes of low phosphorous (LP) tolerance. Consequently, the exploration and utilization of LP-tolerant genes are of great significance in plants. The maize inbred line 178 is an inbred LP-tolerant line. In the current study, the expression of this inbred line was induced under the stress of LP conditions. We applied cDNA-amplified fragment length polymorphism to screen LP-tolerant genes and obtained and sequenced 78 differentially expressed gene fragments. Their functions were predicted via bioinformatic analysis. There were no function annotations for 8 differentially expressed fragments. Nine genes exhibited high homology to Arabidopsis thaliana and Oryza sativa genes involved in phosphorus metabolism. This study lays a good foundation for further cloning and verification of the genes
\end{abstract}


involved in phosphorus metabolism in maize.

Key words: Low phosphorus; Tolerance; Function annotation; Amplified fragment length polymorphism; Maize

\section{INTRODUCTION}

Phosphorous is the second most important element that influences plant growth and development, comprising approximately $0.05-0.5 \%$ of the gross dry weight in plants (Vance et al., 2003). It not only is a component in many important organic compounds (nucleic acids, protein, lecithin, and auxin) in organisms but also is involved in various physiological and biochemical processes via many pathways (Plaxton, 1996). In the natural environment, plants absorb phosphorous in the forms of $\mathrm{HPO}_{4}{ }^{2-}$ and $\mathrm{H}_{2} \mathrm{PO}_{4}^{-}$. The total phosphorus content in the soil is relatively high, but little of it can be absorbed and used directly by plants. This is known as typical phosphorus deficiency (Epstein, 1998).

Different plants adapt differently to adverse situations. Some plant species or genotypes develop stronger abilities to absorb and utilize phosphorus in the soil (Lambers et al., 2006) and can obtain higher live weights in conditions of lower phosphorus supply than other species or genotypes (Miller et al., 2003). The differences in the low phosphorus (LP) tolerability of different species, varieties, and strains indicate that screening to identify genotypes with high phosphorus efficiency is a plausible venture (Vance, 2001). Phosphorus-efficient genes can generate increased function via the regulation of their own reaction mechanisms (Hammond et al., 2003). Genetic analysis and cross experimentation indicate that important genes control root growth related to phosphorus nutrition and phosphorus absorption, accumulation, and utilization (Uhde-Stone et al., 2003). These features are inherited and belong to quantitative traits that are controlled by polygenes. Moreover, the expression of these genes is relatively complicated (Yi et al., 2005). Therefore, attempting to acquire species with high phosphorus efficiency via normal hybridization methods appears unpromising. The essence of the differences in phosphorus nutrition genotypes remains unknown. Hence, exploring new LP-tolerant genes and understanding the mechanism of LP tolerance are significantly important endeavors.

The phosphorus-regulated genes found in plants include PHR1 (Arabidopsis thaliana) and PTF1 [A. thaliana, Oryza sativa (rice)], amongst others (Yi et al., 2005; Nilsson et al., 2007). PHR1 belongs to the MYB family of transcription factors, which is homologous to PSRI in Chlamydomonas. PSRI is a transcription factor that is related to the phosphorus reaction, while PHR1 is an activating transcription factor that is downstream of the phosphatemediated signal transduction pathways that influence a series of phosphate starvation response genes. PHRI is located in the nucleus and binds the P1BS conserved sites in the promoter regions of phosphorus response genes. Using a gene chip, Wu et al. (2003) identified several types of transcription factors that may participate in the regulation of the prophase of phosphorus starvation responses.

This study used cDNA-amplified fragment length polymorphism (cDNA-AFLP) to identify candidate LP-tolerant genes in the Zea mays (maize) inbred line 178, which can be produced under stressed and unstressed conditions. The differentially expressed fragments were isolated and cloned for bioinformatic analysis. This study provides the theoretical basis for further research on the cloning of related genes, the characteristics of gene expression, and the development of new molecular makers. 


\section{MATERIAL AND METHODS}

\section{Cultivation of maize under LP conditions}

The soil used for the maize plants was washed 3 times with deionized water to remove any inorganic salts contained in the impurities and soil particles. The soil was homogenized, sieved (2-mm mesh), and autoclaved at $120^{\circ} \mathrm{C}$ for $30 \mathrm{~min}$ to prevent the influence of microorganisms on seed germination. Seeds germinating after 2 days of imbibition were planted in 2 pots ( 30 seeds per pot) containing the autoclaved soil that had been supplemented with Hoagland LP nutrient solution and normal nutrient solution, respectively. The fresh leaves and roots from maize seedlings at the 4-leaf stage were extracted for total RNA isolation.

\section{Screening relevant genes by cDNA-AFLP}

Total RNA was extracted from the roots and leaves obtained under LP-stressed and -unstressed conditions using RNAiso Plus reagent (TaKaRa Biotechnology Co., Ltd., Dalian, China). The quality of the RNA was checked by denaturing formaldehyde agarose gel electrophoresis and ethidium bromide. Double-stranded (ds) cDNAs were prepared using the components and procedures of the SMART ${ }^{\mathrm{TM}}$ PCR cDNA Synthesis Kit (Clontech, Mountain View, CA, USA). The following cDNA-AFLP joints and primers were synthesized by the Shanghai Sangon Company (Shanghai, China): pre-amplification primer E, 5'-GACTGCGTACCAATT C-3'; pre-amplification primer M, 5'-GATGAGTCCTGAGTAA-3'; selective primer E, 5'-GACTGCGTACCAATTCNN-3'; and selective primer M, 5'-GATGAGTCCTGAGTAANN-3'.

The selective primers were extended by 2 bases $(+2)$ at the 3 '-end of the universal primer U. Thus, selective primer $\mathrm{E}+2$ would be GACTGCGTACCAATTCNN (N could correspond to A, $\mathrm{C}, \mathrm{G}$, or T), and selective primer $\mathrm{M}+2$ would be GATGAGTCCTGAGTAANN (N could correspond to A, C, G, or T) in some of the sets of designed primer pairs. This study selectively extended 2 bases of maize cDNA; 16 sets of selective primer $\mathrm{E}$ and 16 sets of selective primer $\mathrm{M}$ were combined to yield 256 primer pair combinations in total. The polymerase chain reaction (PCR) products were assayed on a Bio-Rad Sequi-Gen ${ }^{\mathrm{TM}}$ sequencing electrophoresis apparatus (Bio-Rad, Hercules, CA, USA), and DNA fragments were visualized by silver staining.

\section{Identification of differentially expressed genes}

We selected the target fragments by polyacrylamide gel electrophoresis (PAGE). We added $50 \mu \mathrm{L}$ sterile $\mathrm{H}_{2} \mathrm{O}$ to the sheared gel pieces, eluted the DNA at $37^{\circ} \mathrm{C}$, and incubated the sample for $10 \mathrm{~min}$ at $100^{\circ} \mathrm{C}$. The specific segments were recycled by PCR. Then, they were ligated into a pMD18-T vector (TaKaRa, Japan). Sequence analysis of the screened positive clones was conducted. The sequence data were deposited in EMBL (http://www.embl.org) and GenBank (http://www.ncbi.nlm.nih.gov) data libraries under accession numbers JZ773723 to JZ773800.

\section{Functional analysis of candidate genes}

To investigate the gene functions and structural domains in maize, gene ontology (GO) annotation and Basic Local Alignment Search Tool (BLAST) analysis were applied. 
Then, we predicted the structural domains in the Pfam database (http://pfam.sanger.ac.uk/ search/sequence) and compared these genes to data from the Arabidopsis Affymetrix ATH1 array to achieve a better understanding of the metabolic and signal transduction pathways involving the proteins that were expressed differentially in the leaves and roots under LP stress conditions. The genes were classified according to the functional category database of A. thaliana (http://mips.gsf.de/proj/thal/db/index.html).

\section{RESULTS}

\section{Comparison of leaflets under normal and LP conditions}

Trefoil-stage leaflets grown in normal phosphorous conditions were compared with those grown in LP conditions. Under LP stress, an obvious change was noted in the shape of the roots: the lateral roots and fibrils were stronger during LP stress. Nevertheless, there was not much change in the leaf shape. Because roots are the major organs of higher plants that absorb mineral elements, they possess a high degree of morphologic plasticity, altering their own architecture to better adapt to the changing external environment (Mou et al., 2012). LP stress caused obvious changes in the root architecture, including the length of the lateral roots and fibrils (Hawkesford et al., 2011). In this way, the contact areas between the roots and the soil are enhanced, thereby improving the absorption efficiency (Whitmore and Whalley, 2009).

\section{Screening of differentially expressed genes}

The RNA purity and integrity were checked by spectrophotometry and electrophoresis (Figure 1A). The PCR products $(5 \mu \mathrm{L})$ from 5 different PCR cycle numbers were identified on an agarose gel (Figure 1B). The ds cDNA synthesized in cycle number 21 was more integrated, and some of the transcribed RNA was detected in abundance in the total RNA (Figure 1C). The ds cDNA of the leaves and roots in the normal phosphorous and LP conditions were digested with EcoRI and MseII enzymes, which could identify 4 bases over $5 \mathrm{~h}$. Samples (10 $\mu \mathrm{L}$ ) were identified using agarose gel electrophoresis (Figure 1C). DNA fragments between 100 and $1000 \mathrm{bp}$ indicated sufficient enzyme digestion. These corresponded to the normal cDNA-AFLP results, and were suitable for further pre-amplification. The products obtained using the pre-amplifying primers were between 100 and $2000 \mathrm{bp}$, corresponding to the result of enzyme digestion. In addition, a high product concentration could be diluted 30-50-fold and serve as a template for further research (Figure 1D).

Thirty-two selective primers were used for the cDNA-AFLP analysis. Differential display analysis of the near-isogenic line of maize in the normal phosphorous and LP conditions was carried out using these primer combinations. The products obtained using the selective primers were resolved by 6\% denaturing PAGE and visualized by silver staining (Figure 2). Advantages to utilizing the differential display analysis in combination with the digested ds cDNA-AFLP analysis and silver staining included high resolution, simplicity, reliability, and high polymorphism (Burger and Botha, 2004). A preliminary study, which aimed to examine the different expression patterns of different amplification products, was evaluated according to the following standards. If the fragments existed or were abundant in the samples obtained from the LP stress conditions but were scarce or not found in samples obtained under normal phosphorous conditions, they were considered LP stress-related up-regulated fragments. If 
the fragments were scarce or nonexistent in samples obtained under LP stress conditions but existed or were abundant in samples obtained under normal phosphorous conditions, they were considered LP stress-related down-regulated fragments. This study used 136 primer pair combinations to carry out systematic analysis of 2 samples. We detected 142 differentially expressed fragments, including 121 LP stress-related up-regulated fragments and 21 LP stressrelated down-regulated fragments.
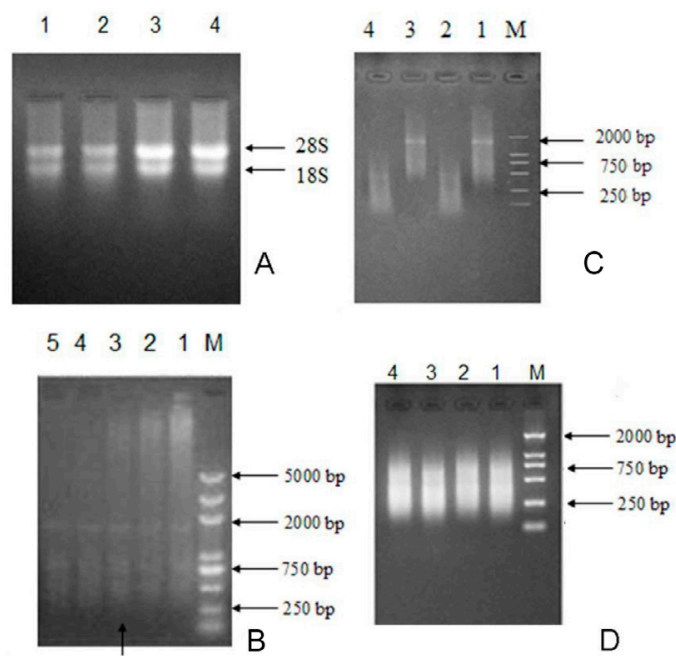

Figure 1. RNA electrophoresis and cDNA synthesis. A. Lane 1, RNA of roots in normal phosphorus conditions; lane 2: RNA of roots in low phosphorus (LP) conditions; lane 3, RNA of leaves in normal phosphorus conditions; and lane 4, RNA of leaves in LP conditions. B. Lane 1, cDNA of cycle 27; lane 2, cDNA of cycle 24; lane 3, cDNA of cycle 21; lane 4, cDNA of cycle 18; and lane 5, cDNA of cycle 27. C. Lane 1, cDNA of roots in normal phosphorus conditions; lane 2, digestion segments of cDNA from normal phosphorus conditions; lane 3, cDNA of roots in LP conditions; and lane 4, digestion segments of cDNA from LP conditions. D. Lane 1, pre-polymerase chain reaction (PCR) products of roots in LP conditions; lane 2, pre-PCR products of roots in normal phosphorus conditions; lane 3, pre-PCR products of leaves in LP conditions; and lane 4, pre-PCR products of leaves in normal phosphorus conditions. Lane $M$, molecular marker.

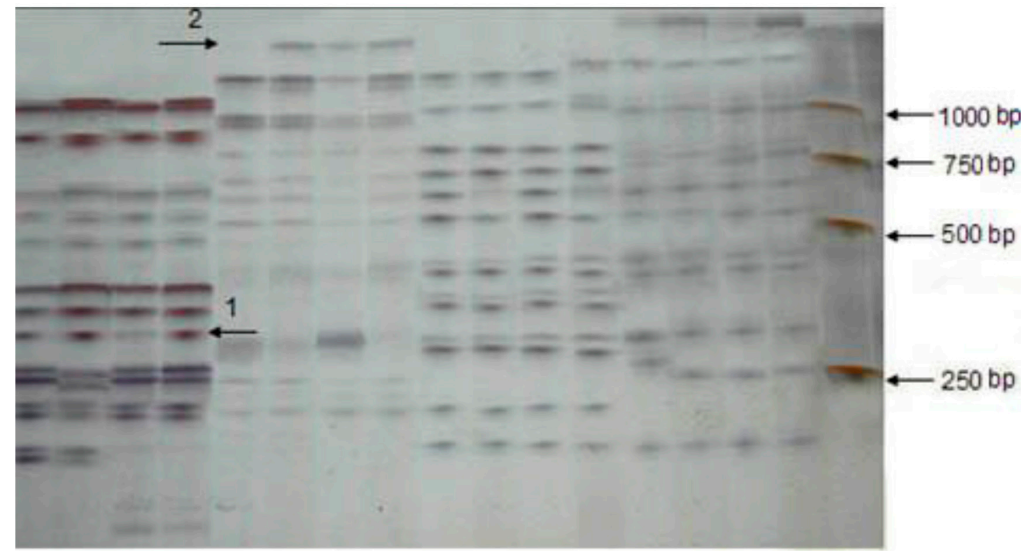

Figure 2.cDNA-amplified fragment length polymorphism products separated by polyacrylamide gel electrophoresis. 


\section{Functional analysis of differentially expressed fragments}

Sequence analysis of the differentially expressed fragments was applied after ligating the fragments into the pMD18-T vector. The sequences of 78 fragments (34 from leaf samples and 44 from root samples) were analyzed successfully. GO analysis was employed to investigate the functions of these differentially expressed fragments (Table 1). A function prediction containing 78 differentially expressed fragments was constructed, allowing us to determine the relationships between the differentially expressed fragments and gene function. We used GO: TermFinder for the bioinformatic analysis. The locations, expressive locations, and corresponding functions of these genes were known. From the functional analysis of the 34 leaves, 3 differentially expressed fragments (P2, P11, and P25) were without annotation and function prediction, 5 differentially expressed fragments (P4, P12, P17, P22, and P33) were unknown proteins, and the remaining 26 fragments were related to multiple metabolic pathways such as energy metabolism, secondary metabolism, signal transduction, protein synthesis, and resistance. Among these differentially expressed fragments, 2 were confirmed as being directly related to phosphorus metabolism: P3 is involved in the mechanism of phosphorus response, and P34 is related to phosphorus metabolism and material synthesis in the plants. The SPX domain, which is annotated to the P3 fragment and is mainly related to the in vivo phosphorus response and metabolism in other plants, is found in both $A$. thaliana and $O$. sativa.

Five differentially expressed fragments (P37, P40, P44, P66, and P73) were not annotated, and their functional description was in the roots; 6 were unknown proteins ( $\mathrm{P} 42$, P46, P53, P57, P61, and P76); and 7 were directly related to phosphorus transport (P58, P60, $\mathrm{P} 63$, P67, P68, P69, and P72). The remaining fragments were related to primary and secondary metabolism, energy metabolism, signal transduction, protein synthesis, and resistance; these functions might have a significant connection with phosphorus. There were many abiotic stress response genes in these specific expression genes, such as $P 4, P 47, P 78$, and $P 36$. Among these, the genes for a glutathione- $S$-transferase $(P 78)$ and a peroxidase $(P 36)$ were expressed specifically, indicating that the above genes exerted significant effects in eliminating reactive oxygen species (ROS) caused by the LP stress and relieving the imbalanced ROS scavenging system in the plants. There was high homology between $P 31$ and the phosphorus transporter PT2, and P31 was expressed specifically under LP stress. This demonstrated that these genes possessed the extraordinary ability to enhance the response of a plant to LP stress and adapt to the stress.

Using BLAST-based methods, we confirmed that 9 genes were directly related to phosphorus circulation, transportation, and response (Tables 2 and 3). Among the 3 genes $(P 34, P 60$, and $P 69)$ participating in phosphorus circulation, $P 34$ had the highest homology ( $80 \%$ ) to $O$. sativa and up to $75 \%$ homology with $A$. thaliana. However, $P 60$ had low homology (below 30\%) with the genes of $O$. sativa and $A$. thaliana.

Of the 3 candidate genes $(P 58, P 63$, and $P 67)$ that participate in relative phosphorus transportation, $P 67$ had $93 \%$ homology with OSIGBSA001P07 of O. sativa. However, P67 had less than $30 \%$ homology with genes of both $O$. sativa and A. thaliana. Of the 3 genes ( $P 3$, $P 68$, and $P 72)$ that participate in phosphorus response, $P 3$ had the highest homology $(83 \%)$ with $A T 5 G 15330$ of $A$. thaliana and $100 \%$ homology with $O$. sativa. However, $P 72$ had low homology (30\%) with $O$. sativa and $A$. thaliana genes. 


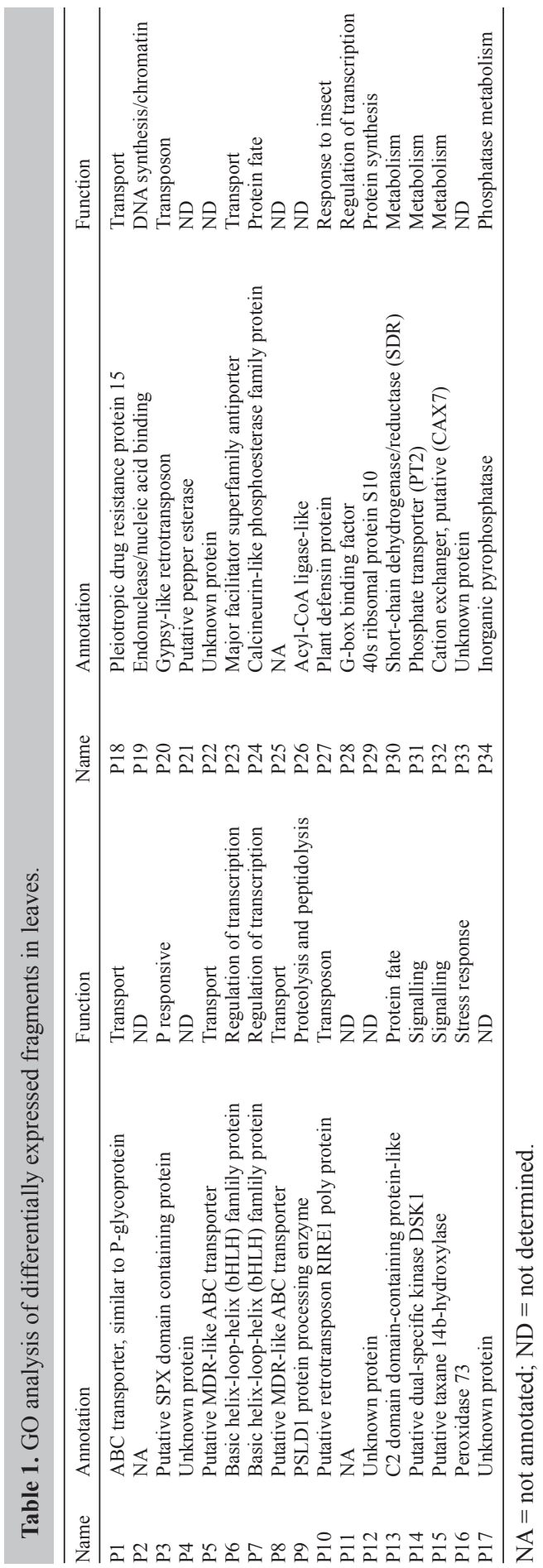


H.Y. Jiang et al.

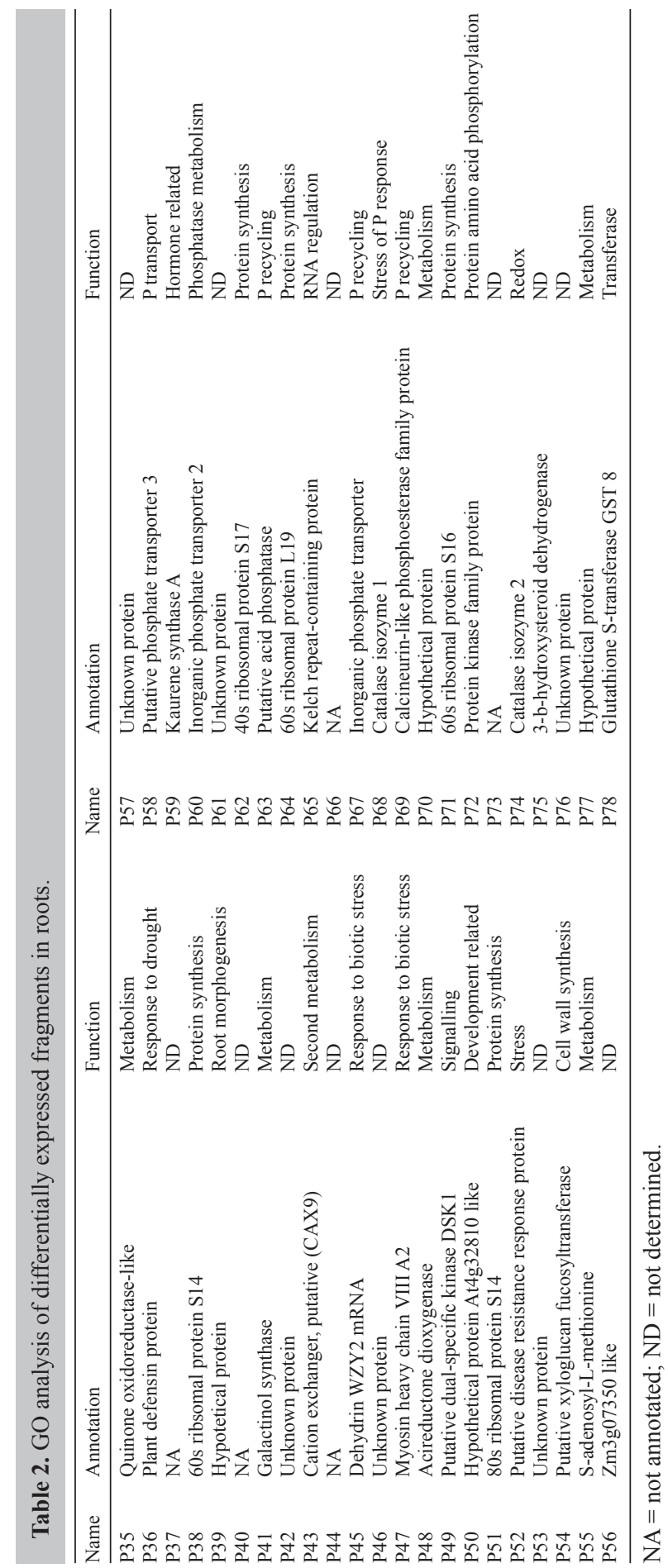


Table 3. Homology analysis of differentially expressed fragments.

\begin{tabular}{llll}
\hline Name & Expressing part & Full-length sequence of maize (bp) & Homology comparison \\
\hline P3 & Leave & (ZM_BFc0172G17) 999 & $\begin{array}{l}\text { AT5G15330 (Arabidopsis thaliana): } \\
\text { Homology }=83 \% \text { J013066K23 (Rice): Homology }=100 \%\end{array}$ \\
P34 & Leave & (LOC100282768) 1001 & AT2G46860 (Arabidopsis thaliana): \\
& & Homology $=75 \%$ OSIGCSA021P07(Rice): Homology $=80 \%$ \\
P58 & Root & (LOC732717) 2096 & None \\
P60 & Root & (LOC100284565) 1176 & None \\
P63 & Root & (ZM_BFc072M11) 1997 & OSJNBb0003A12 (Rice): Homology $=89 \%$ \\
P67 & Root & (ZM_BFb0100H13) 1770 & OSIGBSA001P07 (Rice): Homology $=93 \%$ \\
P68 & Root & (LOC 801284655$) ~ 2031$ & J013145C05 (Rice): Homology $=96 \%$ \\
P69 & Root & (pco091663a) 1667 & Os09g0533300 (Rice): Homology $=86 \%$ \\
P72 & Root & (LOC300074) 1096 & None \\
\hline
\end{tabular}

\section{Function classification of differentially expressed proteins}

To further understand the different proteins involved in the metabolic and signaling pathways in low and normal phosphorus conditions, GO analysis of 70 proteins were used for function classification (Figure 3). The graph is divided into 9 categories: unknown protein function $(12 \%)$, cell cycle/transport $(6 \%)$, protein synthesis $(8 \%)$, cell rescue/defense/toxicity protein related $(8 \%)$, secondary metabolism $(5 \%)$, translation and signal transmission mechanism (10\%), energy metabolism (12\%), metabolism related (33\%), and protein fate $(6 \%)$. The majority of the proteins correlated to metabolic functions, and differences between the leaf and the root demonstrate that a plant utilizes multiple means of adaptation to an LP environment, controlling phosphorus absorption and transformation.

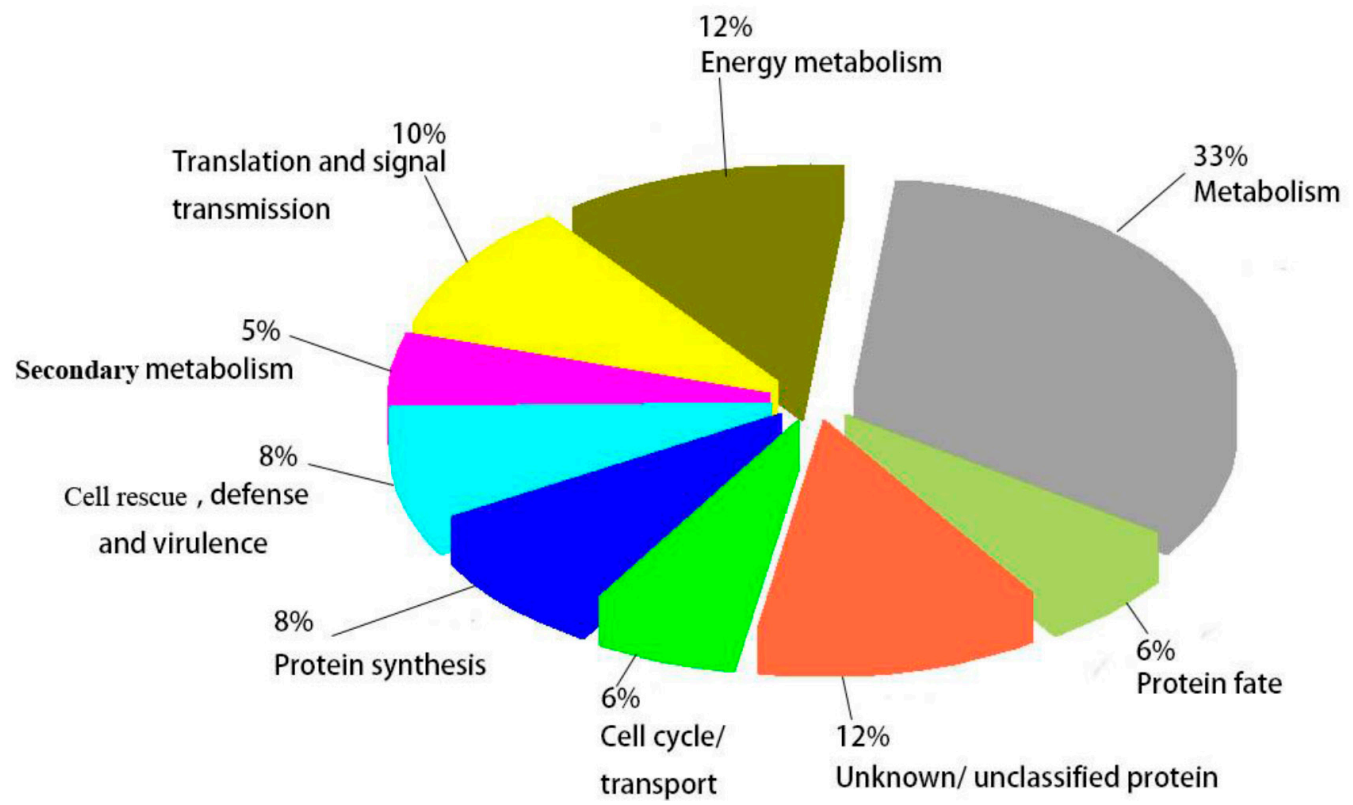

Figure 3. Function classification of differentially expressed proteins. 


\section{DISCUSSION}

The maize inbred line 178 is a type of LP-tolerant inbred line (Hao et al., 2008). Gene expression is characterized by time-space associations. Thus, selection of the proper period and material is the key to successfully obtaining target genes. In maize, the differentiation of the leaves and roots is highly active during the period of germination to the trefoil stage. The root is the main tissue for most LP gene cloning, which provides a foundation for the successful separation of differentially expressed fragments from plants grown under LP stress (Hernández et al., 2007).

Among the LP stress-related up-regulated genes, several are involved in the degradation and synthesis of proteins. In plants, LP tolerance involves an extremely complicated process, and its mechanism relates to each stage of the process. Consequently, there is an obvious change in the protein content in the plant cell when subjected to LP stress. However, the relevant mechanisms regarding the generation of inducible specific proteins under LP stress and the mechanism of response to phosphorus stress remain unclear. Many studies have demonstrated that the gene expression patterns of plants change distinctly (Hammond et al., 2003; Wen et al., 2009; Wang et al., 2011, Cai et al., 2013). In addition, LP situations induce the synthesis of new proteins. By responding to phosphorus stress, these proteins participate in the physiological and biochemical processes of the plants (Dakora and Phillips, 2002).

Under LP stress, the homology analysis of 9 genes directly related to transportation, circulation, and transduction demonstrated that each gene had high similarity to the isolated mRNA of maize. This means that the cDNA-AFLP involving the double digestion of cDNA by 2 restriction endonucleases was suitable for the study of the differentially expressed genes in maize (Fusco et al., 2005) and that the differentially expressed fragments we isolated were reliable. Among these, $P 3$ and $P 34$ had high homology with $A$. thaliana and $O$. sativa; $P 67$ had high homology with A. thaliana and $O$. sativa; and $P 63, P 68$, and $P 69$ had high homology with $O$. sativa. In these species, there was similarity in the structural domains of these genes, and these genes acquired the same functions under LP conditions. In contrast, the homology analysis showed that $P 58, P 60$, and $P 72$ had no more than $30 \%$ homology with $A$. thaliana or $O$. sativa genes. This evidence indicates that these 3 genes are possibly unique to maize, which is of great significance in the study of the molecular and response mechanisms under LP conditions.

\section{ACKNOWLEDGMENTS}

We extend our thanks to the reviewers for their careful reading and helpful comments. Research supported by the Natural Science Foundation of China (\#313013241, \#31371285) and the Scientific and Technological Research Plan of Anhui (\#1206c0805032,\#KJ2011A110).

\section{REFERENCES}

Burger AL and Botha FC (2004). Cloning of a specific ripening-related gene from the multiple of ripening-related genes identified from a single band excised from a cDNA-AFLP gel. Plant Mol. Biol. Rep. 22: 225-236.

Cai H, Xie W and Lian X (2013). Comparative analysis of differentially expressed genes in rice under nitrogen and phosphorus starvation stress conditions. Plant Mol. Biol. Rep. 31: 160-173.

Dakora FD and Phillips DA (2002). Root exudates as mediators of mineral acquisition in low-nutrient environments. Plant Soil 245: 35-47.

Epstein E (1998). Crops tolerant of salinity and other mineral stresses. In: Better crops for food. Ciba Foundation 
Symposium 97, London, 61-68.

Fusco N, Micheletto L, Dal Corso G, Borgato L, et al. (2005). Identification of cadmium-regulated genes by cDNA-AFLP in the heavy metal accumulator Brassica juncea L. J. Exp. Bot. 56: 3017-3027.

Hammond JP, Bennett MJ, Bowen HC, Broadley MR, et al. (2003). Changes in gene expression in Arabidopsis shoots during phosphate starvation and the potential for developing smart plants. Plant Physiol. 132: 578-596.

Hao L, Zhang J, Chen F, Christie P, et al. (2008). Response of two maize inbred lines with contrasting phosphorus efficiency and root morphology to mycorrhizal colonization at different soil phosphorus supply levels. J. Plant Nutr. 31: 1059-1073.

Hawkesford MJ and Barraclough P (2011). The molecular and physiological basis of nutrient use efficiency in crops. Wiley-Blackwell, Hoboken.

Hernández G, Ramírez M, Valdés-López O, Tesfaye M, et al. (2007). Phosphorus stress in common bean: root transcript and metabolic responses. Plant Physiol. 144: 752-767.

Lambers H, Shane MW, Cramer MD, Pearse SJ, et al. (2006). Root structure and functioning for efficient acquisition of phosphorus: matching morphological and physiological traits. Ann. Bot. 98: 693-713.

Miller CR, Ochoa I, Nielsen KL, Beck D, et al. (2003). Genetic variation for adventitious rooting in response to low phosphorus availability: potential utility for phosphorus acquisition from stratified soils. Funct. Plant Biol. 30: 973985.

Mou P, Jones RH, Tan Z, Bao Z, et al. (2012). Morphological and physiological plasticity of plant roots when nutrients are both spatially and temporally heterogeneous. Plant Soil 364: 373-384.

Nilsson L, Müller R and Nielsen TH (2007). Increased expression of the MYB-related transcription factor, PHR1, leads to enhanced phosphate uptake in Arabidopsis thaliana. Plant Cell Environ. 30: 1499-1512.

Plaxton WC (1996). The organization and regulation of plant glycolysis. Ann. Rev. Plant Physiol. Plant Mol. Biol. 47: $185-214$.

Uhde-Stone C, Zinn KE, Ramirez-Yáñez M, Li A, et al. (2003). Nylon filter arrays reveal differential gene expression in proteoid roots of white lupin in response to phosphorus deficiency. Plant Physiol. 131: 1064-1079.

Vance CP (2001). Symbiotic nitrogen fixation and phosphorus acquisition: plant nutrition in a world of declining renewable resources. Plant Physiol. 127: 390-397.

Vance CP, Uhde-Stone C and Allan DL (2003). Phosphorus acquisition and use: critical adaptations by plants for securing a nonrenewable resource. New Phytol. 157: 423-447.

Wang S, Jiang J, Li T, Li H, et al. (2011). Influence of nitrogen, phosphorus, and potassium fertilization on flowering and expression of flowering-associated genes in white birch (Betula platyphylla Suk.). Plant Mol. Biol. Rep. 29: 794-801.

Wen F, Woo HH, Pierson EA, Eldhuset TD, et al. (2009). Synchronous elicitation of development in root caps induces transient gene expression changes common to legume and gymnosperm species. Plant Mol. Biol. Rep. 27: 58-68.

Whitmore AP and Whalley WR (2009). Physical effects of soil drying on roots and crop growth. J. Exp. Bot. 60: 28452857.

Wu P, Ma L, Hou X, Wang M, et al. (2003). Phosphate starvation triggers distinct alterations of genome expression in Arabidopsis roots and leaves. Plant Physiol. 132: 1260-1271.

Yi K, Wu Z, Zhou J, Du L, et al. (2005). OsPTF1, a novel transcription factor involved in tolerance to phosphate starvation in rice. Plant Physiol. 138: 2087-2096. 